\title{
Autorregulación, percepción de riesgo y autoexploración de los senos para detección temprana del cáncer de mama: estudio exploratorio-descriptivo
}

\author{
Alicia Saldívar-Garduño \\ Universidad Autónoma Metropolitana, México \\ Fredi Everardo Correa-Romero \\ Universidad de Guanajuato, México
}

Recibido: 5 de junio del 2013 / Aprobado: 11 de julio del 2013

Se examina la relación entre la autorregulación de la conducta, la percepción de riesgo y la autoexploración de los senos como una forma de conocer algunos de los factores que determinan conductas de autocuidado de la salud, como una estrategia que permite detectar el cáncer mamario en fases tempranas y con ello aumentar la probabilidad de su atención oportuna y la esperanza de vida de las mujeres. Se trabajó con una muestra de 467 mujeres entre los 18 y los 65 años, provenientes de la Ciudad de México y municipios conurbados. Los resultados indican que la autorregulación está conformada por dos factores, los cuales muestran valores más altos cuando las mujeres perciben un mayor riesgo de padecer cáncer y cuando se han realizado previamente exploraciones de seno.

autorregulación de la conducta / percepción de riesgo / autoexploración de los senos / cáncer de mama

Self-regulation behavior, risk perception and breast self-exploration as a strategy for early breast cancer detection: an exploratory-descriptive study

The present study explores the relationship between self-regulation of behavior, risk perception and self-examination of the breasts, as a way to know some of the factors that determine behavior to detect breast cancer in its early stages and thus increase the likelihood of timely care and life expectancy of women. The study sample consisted of 467 women between 18 and 65 years, from Mexico City and neighboring municipalities. The results indicate that self-regulation includes two factors: self-oriented early detection of tumors and self-oriented planning and conduct of attention, both of them show higher values when women perceive a greater risk of cancer and when they have previously performed breast examinations.

self-regulation of behavior / risk perception / breast self-examination / breast cancer

Correo electrónico: asaldivarg@gmail.com 


\section{INTRODUCCIÓN}

Actualmente, en el mundo muere una cantidad considerable de personas por diferentes modalidades de cáncer, y ese número se incrementa en forma constante con el paso del tiempo. El cáncer ocupa, hoy en día, el segundo lugar entre las causas de muerte, tanto en los países desarrollados como en los denominados «en transición»y afecta a toda clase de personas, sin distingo de edad, situación socioeconómica, sexo, etcétera, impactando no solo a quienes lo padecen, sino a sus familias y a las sociedades en su conjunto.

A pesar de los significativos avances científicos en la materia, aún existe una gran cantidad de dudas acerca de las causas que desencadenan la aparición de un tumor maligno y el desarrollo del cáncer, pero se conocen un conjunto importante de factores de riesgo para las personas en general. La American Cancer Society (2009) define como factor de riesgo cualquier cosa que aumente las probabilidades de que una persona padezca una determinada enfermedad, en este caso, cáncer. Estos factores se pueden agrupar, de acuerdo con la Organización Mundial de la Salud (OMS, 2009), en estilos de vida, exposición a carcinógenos en el medio ambiente y en el entorno laboral, exposición a formas variadas de radiación o de productos químicos empleados en las industrias y haber padecido algunas infecciones (por ejemplo, hepatitis B o virus del papiloma humano).
Entre los factores que se han identificado como asociados con el cáncer mamario se pueden mencionar la menarca temprana (antes de los 12 años), la menopausia tardía (después de los 50 años), no haberse embarazado nunca o haberlo hecho hasta después de los 30 años, no haber lactado nunca, la obesidad, así como dietas con un alto contenido de grasas, ingestión de bebidas alcohólicas, uso prolongado de anticonceptivos hormonales orales, antecedentes directos de familiares con ese tipo de cáncer (especialmente la madre y las hermanas), entre otros (Instituto Nacional de Cancerología [Incan], 2009; Padilla \& Vallejo, 1999).

A pesar de ello, existen datos que indican que una gran cantidad de las pacientes que llegan a desarrollar cáncer en alguno de los senos no tenían factores de riesgo (especialmente una familiar consanguínea cercana que lo hubiera padecido). Esta información revela que es muy complicado predecir la aparición del cáncer mamario, lo que dificulta su prevención, razón por la cual una proporción importante de las mujeres que acuden a una consulta para una revisión de las mamas se encuentran en etapas avanzadas de la enfermedad, eso reduce en forma considerable su esperanza de vida. Por ello, aunque el riesgo de padecer cáncer de mama es mayor en los países desarrollados, la probabilidad de morir por esa causa aumenta si se vive en los países en desarrollo, también llamados «en transi- 
ción» (OMS, 2009). Se estima que en estos lugares solo el $6,5 \%$ de los casos tienen esperanza de curación completa (Vega, 2010); como dato complementario, se sabe que los casos detectados en la etapa más temprana tienen hasta $98 \%$ de probabilidad de supervivencia durante los primeros 5 años (Hidalgo, Pérez \& Villanueva, s/f).

Por todo lo anterior, las principales estrategias de intervención tienen que ver con la detección del cáncer en fases tempranas, con lo cual aumenta ampliamente la probabilidad de que se evite su avance y diseminación por otros órganos del cuerpo, reduciendo el número de personas, mayoritariamente mujeres, que mueren por esa causa.

Algunos especialistas en el tema consideran que aparte de la escasa educación para el cuidado de la salud, de la deficiente información de que se dispone en México con relación al padecimiento, y además de lo costosas que resultan las pruebas como la mastografía, hace falta sensibilizar a la población femenina del riesgo que existe de padecer esta enfermedad, así como de la necesidad de realizarse periódicamente una autoexploración visual y manual, una consulta médica cada año y, desde los 40 años, una mastografía con la misma frecuencia.

Un dato relevante es que, a pesar de conocer esta información, en un número importante de estudios se reporta que las mujeres presentan una modera- da o baja percepción de riesgo personal a desarrollar padecimientos graves. La percepción de riesgo personal respecto del cáncer de mama se entiende, en este estudio, como la representación cognoscitiva que tenían las mujeres respecto de la probabilidad de padecer cáncer de mama (Saldívar, 2009).

En otras palabras, es comprensible que una gran cantidad de casos con cáncer se diagnostiquen en etapas avanzadas, pues entre las mujeres $-\mathrm{y}$ también entre los hombres- no existe una práctica generalizada de la autoexploración desde la juventud y menos aún de la realización de la mastografía, que permite detectar tumores del tamaño de un grano de arena a partir de los 40 años (Rodríguez, 2005).

En el presente estudio se exploró la autorregulación como un elemento cognoscitivo que se asocia con el control del propio comportamiento, aspecto que es determinante en conductas específicas como puede ser la realización de la autoexploración de los senos para detectar oportunamente el cáncer de mama. Esta variable hace referencia a una capacidad inmensamente adaptativa que resulta útil a todas aquellas disciplinas que buscan promover la salud física de las personas, el bienestar psicológico y el desempeño en el trabajo (Kuhl, Kazén \& Koole, 2006). Autores como Mischel y Shoda (1995) consideran que la autorregulación, en tanto mecanismo de hábito y control personal de 
la conducta, tiene efectos de largo plazo sobre el desarrollo; el mismo Bandura (2004) postula que los hábitos de salud no cambian por actos de voluntad, sino que se requiere contar con habilidades de motivación y autorregulación.

De acuerdo con Bandura (1991) este constructo se refiere a los procesos por los cuales la psique humana ejerce control sobre sus funciones, estados y procesos internos; se encuentra relacionado con la autoobservación que provee la información necesaria para establecer metas realistas, así como evaluar nuestro progreso para conseguirlas. Posteriormente hay un nivel de desarrollo de autocontrol que está dado por las influencias sociales y la retroalimentación del contexto. Finalmente, el último nivel se alcanza con las creencias de autoeficacia que determinan un sistema anticipatorio proactivo, que puede llevar a un comportamiento sistemático y sostenido en la práctica (Bandura, 2012).

En resumen, de acuerdo con Bandura (1991) la autorregulación consiste en controlar nuestro propio comportamiento e involucra tres mecanismos:

- La autoobservación de nuestras propias conductas, sus determinantes y sus efectos;

- hacer juicios de nuestros actos en relación con un estándar personal o circunstancias ambientales; $y$,

- una autorrespuesta.
Para Fierro Bardají (2002) la autorregulación es el último segmento de un proceso más amplio que incluye diversas actividades cognoscitivas (involucramiento, metas, motivación intrínseca, etcétera) que se gestan en un contexto social (la familia, la escuela, y la sociedad en general). Para nuestro problema, la percepción de riesgo se convierte en un factor de involucramiento que puede crear metas de salud que activan las conductas de autorregulación (en este caso, para evitar la enfermedad). Carecer de percepción de riesgo inhibe la necesidad de que la persona pueda regular su conducta. Por otro lado, la conducta de autoexploración permite observar el propio comportamiento, reforzarlo y alcanzar las creencias de eficacia. No tener conductas de autoexploración rompe el ciclo y las creencias que se pueden formar son de incapacidad. De acuerdo con Schunk (2012), cuando una persona se percibe como incapaz de realizar una tarea, suele rechazar dicha actividad, justificando su comportamiento con una actitud negativa o bien minimizando su importancia.

El objetivo de esta investigación fue explorar las relaciones entre la autorregulación y la percepción del riesgo personal con la práctica rutinaria de la autoexploración de los senos de un grupo de mujeres de la Ciudad de México. Además, se describe el comportamiento de las variables considerando la edad, 
la escolaridad y el nivel de conocimientos de la muestra.

MÉTODO

\section{Participantes}

Las participantes fueron 467 mujeres entre los 18 y los 65 años $(M=31$ años; $\mathrm{DE}=11)$, elegidas por muestreo intencional, de la ciudad de México y municipios conurbados, con el único criterio de que fueran mayores de 18 años. Su estado civil era, principalmente, solteras o divorciadas $(54,2 \%, \mathrm{n}=253)$, casadas o unidas, $45,7 \%(\mathrm{n}=212)$; viudas, $1,5 \%(\mathrm{n}=7)$; y en alguna otra condición, $0,6 \%(\mathrm{n}=3)$; el nivel educativo reportado fue sobre todo básico o medio (55,3 $\%, \mathrm{n}=252$ ), aunque el rango abarcó desde primaria hasta el posgrado, y se dedicaban principalmente a desarrollarse como estudiantes $(27,4 \%, n=116)$ y empleadas $(19 \%, \mathrm{n}=80)$.

\section{Instrumento}

Se usó la «Escala de factores psicosociales asociados con el cáncer de mama en mujeres mexicanas» (Saldívar, 2009), la cual constaba de varias subescalas, que midieron, entre otros factores, la percepción del riesgo personal para desarrollar cáncer de mama en mujeres (estimado con una escala de probabilidad entre 0 y 100), la autorregulación relativa al cuidado de la salud mamaria y la práctica rutinaria de autoexploración de los senos (se les preguntó si alguna vez se habían realizado la autoexploración, si lo habían vuelto a hacer y con qué regularidad la efectuaban).

\section{Procedimiento}

El contacto con las participantes se estableció en diversos escenarios, tales como escuelas, oficinas, hospitales, parques, centros comerciales, etcétera, de la Ciudad de México y regiones aledañas del Estado de México; una vez ubicadas, se les explicó el objetivo de la investigación y se les invitó a colaborar en forma voluntaria, garantizando la confidencialidad y el anonimato de su información. De ser necesario, las preguntas les fueron leídas y sus respuestas registradas en los cuestionarios por una encuestadora o encuestador. Al finalizar, se agradeció a las personas su cooperación con el estudio. Esta aplicación se realizó entre los meses de agosto y septiembre del año 2007.

\section{Análisis}

La información obtenida fue sometida a un análisis de frecuencias para verificar la limpieza de la base de datos y para realizar la validación de los reactivos. A continuación se corrieron análisis descriptivos para conocer la forma de la distribución de las respuestas; el análisis factorial permitió reducir el conjunto total de las variables de la escala de autorregulación a un número menor de dimensiones; para el análisis de con- 
fiabilidad se aplicó la fórmula alfa de Cronbach. Para determinar el impacto de la edad, escolaridad y el nivel de conocimientos, se crearon grupos para hacer comparaciones de los promedios a través de la prueba $\mathrm{t}$ - Student.

\section{Resultados}

Al indagar sobre las prácticas de las participantes respecto de la autoexploración rutinaria de los senos, un 62 $\%(n=267)$ reportó haberse revisado alguna vez, mientras el $38 \%$ restante $(\mathrm{n}=165)$ admitió no realizarla en forma sistemática (casos perdidos $=35$ ).

La percepción de riesgo personal para padecer cáncer de mama se midió con la pregunta «En una escala del 0 a 100 , ¿qué tan probable es que usted desarrolle cáncer de mama si no se autoexplora los senos?». Los resultados obtenidos arrojaron una media de 54 con una D.E. $=26$, lo que indica que ponderaron en un nivel medio la probabilidad de padecer cáncer, a pesar de ello no hay casos que consideren altamente probable padecer cáncer mamario, pues ninguna participante reportó una probabilidad superior al $80 \%$. Además, se hicieron preguntas similares con mujeres cuya relación con las participantes era desde muy cercana, como una mujer de su familia, hasta las más lejanas (cualquier mujer). Como puede apreciarse en el gráfico 1, entre más lejana es la relación con las mujeres de referencia, mayor es el riesgo adjudicado a estas y entre más cercanas, menor es el promedio respectivo.

Al realizar una comparación de los promedios obtenidos en esta variable por grupos de edad, nivel escolar y ni-

\section{Gráfico 1}

Percepción de riesgo de desarrollar cáncer de mama

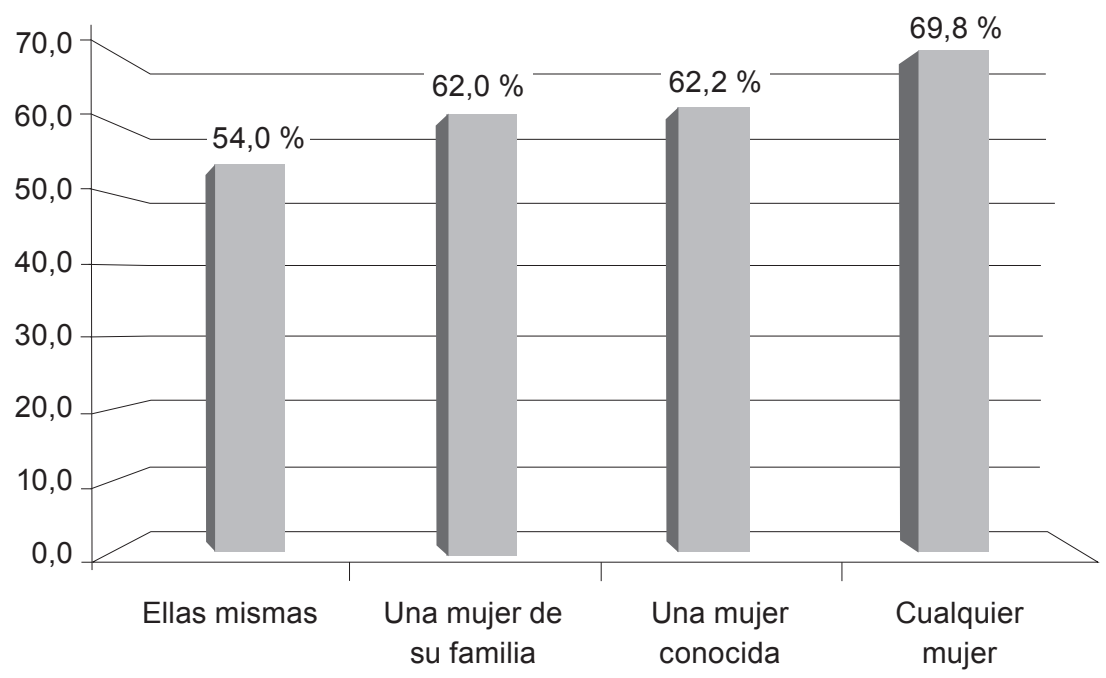


Tabla 1

Comparación de promedios de la percepción del riesgo personal por edad, escolaridad y nivel socioeconómico

\begin{tabular}{llccc}
\hline \multicolumn{1}{c}{ Variable } & \multicolumn{1}{c}{ Grupos } & N & Media & DE \\
\hline Edad & 18 a 28 años & 229 & 52.6 & 26.5 \\
& 29 a 65 años & 160 & 55.9 & 25.3 \\
\multirow{2}{*}{ Nivel escolar } & Básica o media & 193 & 55.3 & 26.7 \\
& Superior & 188 & 53 & 24.9 \\
\multirow{2}{*}{ Nivel de } & Ausente o bajo & 173 & 52.7 & 26.6 \\
conocimientos & Medio o alto & 216 & 55 & 25.5 \\
\hline
\end{tabular}

vel de conocimientos sobre el cáncer de mama, el resultado no reveló diferencias estadísticamente significativas entre estos en ninguno de los casos, aunque sí se observó una percepción de riesgo distinta, como se puede apreciar en la tabla 1 :

La tabla 1 muestra una tendencia: las mujeres cercanas a los 30 años de edad y mayores percibían un riesgo también mayor de padecer cáncer de mama, así como las que contaban con nivel de educación básica o media. Como era de esperarse, con un mayor nivel de conocimientos hay una disposición también mayor para percibir el riesgo.

Por otra parte, la escala de autorregulación orientada al cuidado de la salud mamaria constaba de 8 reactivos que evaluaban el comportamiento relativo a la autoexploración de los senos, las implicaciones que tendría el realizársela o no, y el estado de la salud mamaria en el futuro; estos fueron sometidos a un análisis factorial exploratorio, el cual arrojó dos factores que en conjunto explicaban el $53 \%$ de la varianza y obtuvieron una confiabilidad general de .78 por la fórmula de alfa de Cronbach (para el primer factor la consistencia interna fue
.75 con cuatro reactivos, mientras que para el segundo factor fue de $.62 \mathrm{con}$ cuatro reactivos).

Como se aprecia en la tabla 2, el primer factor contiene reactivos que se refieren sobre todo al propósito de la autoexploración, que es el de detectar tempranamente los nódulos o protuberancias en los senos que pueden ser síntomas de cáncer, razón por la cual se le denominó Autorregulación orientada a la detección de tumores; este primer factor explica el $40 \%$ de la varianza y tiene una confiabilidad de .75. El segundo factor contenía reactivos propios de la planificación de la autoexploración o de visitas al médico con el propósito de realizar la revisión de los senos, lo que da sentido a su etiqueta: Autorregulación orientada a la planificación y conductas de tratamiento. La proporción de la varianza explicada fue de $13 \%$, con una confiabilidad de .62, que puede considerarse bajo, aunque habría que tener en cuenta que se encuentra formado por pocos reactivos.

Al sumar los puntajes de los reactivos incluidos en cada uno de los factores, se obtuvieron dos nuevas variables 


\section{Carga} factorial
Corr. ítem-total

Factor 1. Autorregulación orientada hacia la detección temprana de tumores

A veces me digo a mí misma que debo explorar mis senos para saber si tengo bolitas o tumores.

Ya es tiempo de que me haga un autoexamen de senos para saber si tengo bolitas o tumores.

A veces me doy ánimos para hacer cosas que me ayudan a cuidar mi salud, como revisarme los senos y comprobar que no tengo bolitas o tumores.

Mejor comienzo ya a planear cuál es el mejor momento para hacerme un autoexamen de senos para descartar posibles síntomas de cáncer de mama.
.799

.750

.732

.589

$4.1 \quad 0.9$

.42

Alfa de Cronbach
$15.8 \quad 8.2$

Factor 2. Autorregulación orientada hacia la planificación y conductas de atención

Después de hacerme la autoexploración de los senos, deberé programar una cita con un doctor para hacerme un examen de mamas y confirmar que lo estoy haciendo bien.

Si no me reviso los senos una vez al mes para detectar bolitas, puedo enfermarme de cáncer de mama.

Cuidar mi salud es importante para mí y por esa razón me voy a revisar los senos para prevenir el cáncer de mama.

.785

.745

.565

Será mejor que marque en un calendario los días de cada mes en que debo hacerme la autoexploración de los senos.

\section{Alfa de Cronbach}

.494

.62

$3.9 \quad 1.0$

.48

.41

$3.7 \quad 1.2$

$4.4 \quad 0.7$

.41

$3.8 \quad 1.0$

.36

Escala de respuestas: 1 = Totalmente en desacuerdo, $2=$ En desacuerdo, $3=$ Ni en acuerdo ni en desacuerdo, $4=$ De acuerdo, $5=$ Totalmente de acuerdo.

que, al ser sometidas a un análisis descriptivo, arrojaron, para el primer factor, una media de 15.7, y el segundo, un promedio de 15.8, ambas variables en un rango de 4 a 20 donde la media teórica fue 12. Al aumentar los valores, también lo hizo la capacidad de llevar a cabo acciones reguladas personalmente, con lo cual se puede decir que la muestra tiende a una moderada autorregulación de su conducta.

A continuación, los puntajes de percepción de riesgo fueron divididos en dos grupos, el de los bajos (entre 0 y 50 puntos), y los altos (entre 51 y 100 puntos). La prueba $\mathrm{t}$ - Student mostró para ambos factores que la autorregulación orientada a la detección de tumores, como la relativa a la planificación y conductas para el cuidado de la salud mamaria fueron mayores en el grupo con percepción de riesgo alta, obteniendo diferencias estadísticamente significativas entre los grupos $(p<.05)$; en otras palabras, a mayor percepción del riesgo existen más conductas autorreguladas. 
Tabla 3

Comparación de promedios en autorregulación por percepción de riesgo

\begin{tabular}{lcccccc}
\hline Variable & Grupos & $\mathbf{n}$ & Media & D.E. & F(gl) & t \\
\hline $\begin{array}{l}\text { Autorregulación orientada } \\
\begin{array}{l}\text { a la detección temprana } \\
\text { de tumores }\end{array}\end{array}$ & Riesgo bajo & 266 & 15.6 & 2.9 & 1.070 & $-1.993^{*}$ \\
\hline $\begin{array}{l}\text { Autorregulación orientada } \\
\text { a la planificación }\end{array}$ & Riesgo alto & 151 & 16.1 & 2.8 & -323.071 & \\
\hline
\end{tabular}

${ }^{*} p<0.05$

Posteriormente, se observó la relación entre acciones reguladas personalmente y la práctica rutinaria de la autoexploración de los senos. En este caso, el puntaje en los dos constructos encontrados en el análisis factorial fue mayor para el grupo de mujeres que habían reportado realizarse la autoexploración de los senos en forma rutinaria, y las diferencias en ambos casos resultaron estadísticamente significativas, con lo que podemos decir que la autorregulación se encuentra asociada con la práctica rutinaria, tal como se muestra en la tabla 4.

\section{CONCLUSIONES}

Lo que aquí se presenta es una primera aproximación para conocer cómo la percepción del riesgo personal de padecer cáncer de mama y la autoexploración de los senos se encuentran asociados con el proceso de autorregulación de la conducta.

En primer lugar podemos decir que las escalas utilizadas en este caso resultaron eficientes, pero requieren de ser mejoradas para aumentar su confiabilidad. Se obtuvieron dos factores: uno referido a «la autorregulación orientada a la detección de tumores»» y otro a la «planificación y conductas

Tabla 4

Comparación de promedios en autorregulación por práctica rutinaria

\begin{tabular}{lcccccc}
\hline Variable & Grupos & $\mathbf{n}$ & Media & D.E. & $\mathbf{F}(\mathbf{g l})$ & $\mathbf{t}$ \\
\hline Autorregulación orientada & No rutina & 165 & 15.4 & 2.7 & 0.067 & $-1.960^{*}$ \\
$\begin{array}{l}\text { a la detección temprana de } \\
\text { tumores }\end{array}$ & Sí rutina & 267 & 16 & 2.9 & $(370.219)$ & \\
Autorregulación orientada & No rutina & 165 & 15.4 & 2.9 & 3.451 & $-2.942^{*}$ \\
a la planificación & Sí rutina & 267 & 16.1 & 2.6 & $(316.015)$ & \\
\hline
\end{tabular}


para el cuidado de la salud mamaria»; como indica Fierro Bardají (2002), la autorregulación se encuentra asociada con aspectos cognoscitivos de motivación (factor uno) y con el alcance de metas (factor dos).

No se encontraron diferencias estadísticamente significativas en la percepción de riesgo por edad, nivel educativo o nivel de conocimientos. No queda clara la razón, aunque es alentador ver tendencias en el sentido esperado con la edad y el nivel de conocimiento, no así con el nivel educativo, pues al aumentar los estudios disminuye el riesgo percibido, aspecto que puede estar ligado a un abandono de intervenciones en dicha muestra por considerar que al contar con educación por encima del promedio nacional, las personas se encuentran más informadas sobre todos los temas, incluyendo el del cáncer mamario y su detección oportuna.

Por otro lado, la literatura reporta que generalmente tendemos a ver el riesgo como algo relativamente ajeno a nosotros. Como se puede apreciar en el gráfico 1, el riesgo que se percibe aumenta conforme se atribuye a una persona con un parentesco más lejano, mientras que el riesgo personal es el menos alto; tal resultado se puede explicar a través del sesgo fundamental de la atribución (Weiner, 2012), en el que se postula que los aspectos negativos de la vida le suelen pasar a «otras» personas. Esta «falsa» seguridad mantiene el equilibrio cognitivo y la autoestima, sin embargo, en esta investigación resulta interesante que la percepción de riesgo personal alcanza una probabilidad mayor del 50 $\%$, lo que la hace cada vez menos ajena, resultado, quizás de la penetración de los mensajes difundidos a través de los medios masivos sobre la importancia de llevar a cabo la revisión mensual.

Los resultados confirman los supuestos teóricos, pues una mayor percepción de riesgo está vinculada con una mayor autorregulación, del mismo modo que se relaciona con la conducta previa de realizar una autoexploración. Estos procesos se explican a través del modelo tríadico de Bandura (2012), donde cada elemento (la conducta y los aspectos personales) son mutuamente influyentes; en otras palabras, una conducta se refuerza con los aspectos cognoscitivos y viceversa, creando un círculo virtuoso.

Lo que se desprende de este estudio, en términos prácticos, es que para alcanzar una mayor autorregulación en lo que se refiere a las conductas asociadas con el cuidado de la salud, como en el caso del cáncer de mama, es necesario hacer conscientes a las personas del riesgo de desarrollar esta enfermedad, pero también es preciso educarlas, entrenarlas y convencerlas de contar con una práctica rutinaria de autoexploración. Al respecto, Bandura (2007) señala que un primer nivel de activación pasa por el «modeling», es decir, observar la conducta de un «modelo» al cual seguir, que en este caso puede ser 
una persona (idealmente otra mujer que muestre cómo realizarse una revisión cuidadosa y correcta de los senos) dentro de la familia o quizás en la escuela.

Ahora bien, como apunta Schunk (2012), la autorregulación solo se gesta en un ambiente de autodeterminación, donde el sujeto tiene libertad de llevar a cabo la conducta. ¿Nuestra sociedad permite este grado de autodeterminación? La influencia del contexto es el tercer elemento del modelo tríadico de Bandura (2012), y queda pendiente el estudio de su influencia; sin embargo, estudios previos (Saldívar, 2013) que investigaron la influencia de las normas sociales indican que es un factor determinante para la toma de decisiones en lo que se refiere al cuidado de la propia salud.

No se cuenta todavía con evidencia suficiente sobre la forma como la autorregulación se asocia de manera específica con algunos aspectos de la vida de las personas, como la percepción del riesgo y la realización de acciones para el cuidado de la salud (autoexploración). Para los temas relacionados con este último aspecto, resulta útil, sin embargo, contar con una herramienta que nos permita entender cómo es que las personas planificamos las acciones que pueden llevarnos a prevenir enfermedades, o bien prever las consecuencias y la afectación sobre la salud por no ejecutar ciertos comportamientos saludables.

Contar con pistas claras sobre el funcionamiento de factores como los estudiados, puede tener un impacto importante en el mediano y el largo plazo para planificar de mejor manera las intervenciones psicosociales dirigidas al mejoramiento de las condiciones de salud de los grupos específicos de mujeres.

El siguiente paso es comprobar el efecto del modeling en la creación de hábitos de autocuidado de la salud, el impacto de las rutinas autorreguladas en las conductas de salud y la influencia social en la percepción de riesgo y en las conductas preventivas para reducir y controlar los factores de riesgo. Todo ello estaría encaminado a crear un modelo que se pueda traducir en una intervención psicosocial eficiente y con resultados positivos en la lucha contra el cáncer de mama.

\section{REFERENCIAS}

American Cancer Society (2009). Multiple primary cancers. Atlanta: American Cancer Society.

Bandura, A. (1991). Social cognitive theory of self-regulation. Organizational Behavior and Human Decision Processes, 50, 248-287.

Bandura, A. (2004). Health promotion by social cognitive means. Health Education \& Behavior, 31(2), 143-164.

Bandura, A. (2007). Psychological modeling: conflicting theories. Chicago: Aldine Transaction.

Bandura, A. (2012). Social cognitive theory. En P. A. M. Van Lange, 
A. W. Kruglanski \& E. T. Higgins (Eds.), Handbook of theories of social psychology, Vol. 1, (pp. 349374). Thousand, California: Sage Publications.

Fierro Bardaji, A. (2002). Personalidad, persona, acción. Un tratado de psicología. Madrid: Alianza Editorial.

Kuhl, J., Kazén, M., \& Koole, S. L. (2006). Putting self-regulation theory into practice: A user's manual. Applied Psychology: An International Review, 55(3), 408-418.

Hidalgo de Quevedo, M., Pérez Luna, I. \& Villanueva, V. (s/f). Presentación sobre la prevención y diagnóstico precoz del cáncer de mama. Organización Senosalud, Venezuela. Recuperado de http://www.senosalud.org Instituto Nacional de Cancerología (Incan) (2009). Infocancer. Síntomas de cáncer de mama. Recuperado de $\mathrm{www}$.infocancer.org. $\mathrm{mx} /$ factoresde-riesgo-cancer-de-mama

Mischel, W. \& Shoda, Y. (1995). A cognitive-affective system theory of personality: Reconceptualizing situations, dispositions, dynamics, and invariance in personality structure. Psychological Review, 102(2), 246-268.

Organización Mundial de la Salud (OMS) (2009). ¿Aumenta o disminuye el número de casos de cáncer en el mundo? Recuperado de http:// www.who.int/features/qa/15/es/index.html.
Padilla, G. \& Vallejo, M. (1999). Cáncer de mama. Práctica médica efectiva. Vol. 1 (1). México, D. F.: Instituto Nacional de Salud Pública, Subsecretaría de Prevención y Control de Enfermedades.

Rodríguez Cuevas, S. A. (2005). Cáncer de mama. Cirugía y cirujanos, 73(006), 423-424.

Saldívar Garduño, A. (2009). Factores que favorecen la prevención de enfermedades y el cuidado de la salud de las mujeres. (Tesis para optar el doctorado en Psicología). No publicada. México: UNAM, Programa de Maestría y Doctorado en Psicología.

Saldívar Garduño, A. (2013). Normas sociales y detección temprana de cáncer de mama: recursos para el autocuidado de la salud. VII Semana Internacional de Psicología Social. Universidad Autónoma Metropolitana Iztapalapa, del 20 al 23 de mayo. México, D. F.

Shunck, D. H. (2012). Teorías del aprendizaje. México, D. F.: Pearson. Vega Saldaña, A. (2010). Situación actual del cáncer de mama en México. Presentación de Power Point. México, D. F.: Fundación Tómatelo a Pecho.

Weiner, B. (2012). An attribution theory of motivation. En P. A. M. Van Lange, A. W. Kruglanski \& E. T. Higgins (Eds.), Handbook of theories of social psychology. Volume 1 ( $\mathrm{pp}$. 135-155). Thousand, CA: Sage Publications. 